\title{
Fourier analyses: A mathematical and geometric explanation
}

\author{
MICHAEL YOST, JR., RICHARD A. COOPER, and FREDERICK J. BREMNER \\ Trinity University, San Antonio, Texas 78212
}

\begin{abstract}
Fourier analyses are used in electrophysiological research to reduce EEG data to an interpretable, analyzable form. This article outlines the mathematical similarities and differences between Fourier transforms and fast Fourier transforms. A geometric explanation of the application of fast Fourier transforms and a Fourier series to theta-band EEG data is also included in this article.
\end{abstract}

There has been renewed interest in time-series analysis of late (Benignus \& Muller, 1982; Bremner, Yost, \& McKenzie, 1982; Glass, Wilson, \& Gottman, 1975). This is because time-series analysis has a broad application from social research to neuropsychology to astronomy. As an example of time-series analysis, we will describe the application of the fast Fourier transform (FFT) equation to EEG data using both a mathematical approach and a geometric approach.

Investigators involved in neuropsychological research have for many years been involved in the analysis of electrical activity of animals' brains. In this research, they place an electrode or electrodes as close as possible to the part of the brain that they are interested in investigating. These electrodes are simply small stainless steel wires that are attached to selected filters and amplifiers. When the neurons in the part of the brain being monitored fire, they induce a voltage in the electrodes. This induced voltage is then filtered, amplified, and recorded on a tape recorder. More specifically, a recording is made of the changing voltage of the induced current over the time period that the induced current is in existence. Let us assume that the greater the number of neurons firing at any one time in the vicinity of an electrode, the higher is the voltage, and that the fewer the number of neurons firing at any one time in the vicinity of an electrode, the lower is the voltage. Typically, the short-term result of a neuropsychological experiment is a series of tape recordings of changing voltages over time or different brain locations for different treatment conditions.

\section{HYPOTHETICAL EXAMPLES}

Let us further assume that a neurophysiological investigator is interested in the number of neurons firing at any time (voltage) and the frequency with which the neurons fire (voltage changes per unit time). To obtain this information, the investigator usually

This research was supported in part by USAFOSR Contracts F49620-79-C-0139 and F33617-78-D-0629-0026.

\section{OPTICAL SPECTRUM}

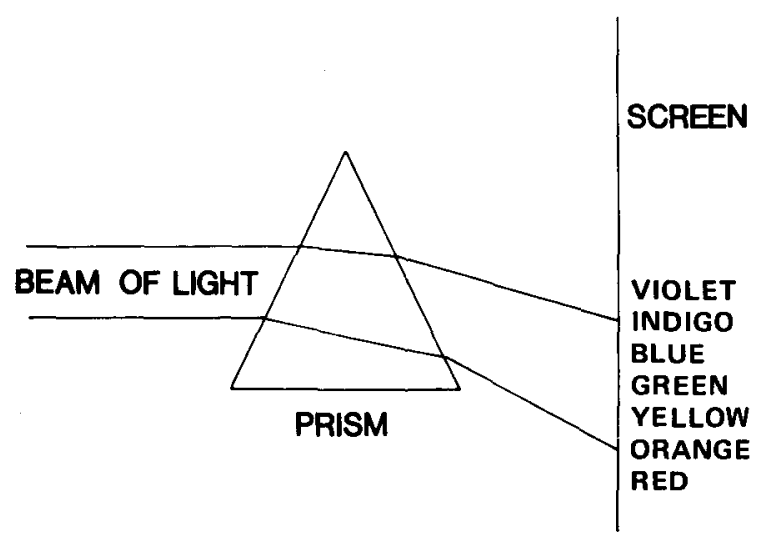

Figure 1. Diagram of white light being transformed by prism into color spectrum.

uses one of two types of Fourier analyses. A Fourier analysis permits an investigator to take the voltage changes that he observed (and recorded) over time and convert these data into usable, analyzable information. That is, a Fourier analysis of data permits an investigator to analyze the original voltage vs. time data in terms of the underlying frequency with which the electrical activity is observed to have occurred under the particular experimental conditions (Walther, 1963). The use of a Fourier analysis in brain research is analogous to the use of a prism in optical research. In optical research, the investigator is interested in analyzing light that is emanating from a source, in order to be able to make some statements about what was happening within the body from which the light originated. To analyze the beam of light, the investigator focuses it on a prism. As is shown in Figure 1, the prism bends the beam of light, breaks it down into its component parts (colors), and displays the colors either on film or on a screen. The investigator then can examine the film or screen to determine which colors are present and in what quantities each color of light is present. Light is a physical phenomenon that conforms to the properties of waves. Each 
color of light has a given frequency, and two or more colors of light emanating from a single source can be separated from each other by using a prism. By examining the frequencies and intensities of light after they have been separated by a prism, an investigator in the field of optics can often make or draw inferences about what was happening within a body from which the beam of light originated. A beam of light in optical research is analogous to the voltage recording in electrophysiological research. Similarly, the prism in the optical research is analogous to the Fourier analysis. The Fourier analysis is a mathematical method of separating the voltage vs. time data to frequency data. The objective of this type of research is to draw inferences about what is happening in the brain by analyzing the frequency data. In the remainder of this article, we will describe how Fourier analysis is used in electrophysiological research.

\section{MATHEMATICAL REPRESENTATION OF FT AND FFT}

The Fourier analysis is carried out in two ways. The Fourier transform (FT) is used to convert mathematical models of voltage vs. time data into frequency data. The FFT is a computation algorithm for the FT and is used to economically convert empirical voltage vs. time models into frequency data. Whereas the FT deals with frequency as a continuous variable, the FFT deals with frequency as a discrete or integer variable. By dealing with frequency as a discrete variable, the data can be coded for analysis into a digital computer (Brigham, 1974). The FFT algorithm greatly reduces the number of calculations and the amount of storage needed to complete the analysis, while the results of the analysis continue to validly represent the electrophysiological activity of the brain. The equation for the FT is:

$$
H(f)=\int_{-\infty}^{\infty} h(t) e^{-j 2 \pi f t} d t
$$

where $h(t)$ is the model of voltage vs. time, $j=-1$, and $\mathrm{e}$ and $\pi$ are constants. Similarly, the equation for the discrete FT is:

$$
H(f n)=\sum_{k=0}^{N-1} h\left(t_{k}\right) e^{-j 2 \pi f_{n} t_{k}}, n=0,1, \ldots, N-1,
$$

where $h\left(t_{k}\right)$ is the voltage at time $t_{k}, H\left(f_{n}\right)$ is the intensity at frequency $f_{n}, N$ is the number of points sampled, $\mathrm{j}=\sqrt{-1}$, and $\mathrm{e}$ and $\pi$ are constants. This can be written in matrix form as:

$$
\overrightarrow{\mathrm{H}}=\mathrm{W}^{\mathrm{nk}} \overrightarrow{\mathrm{h}},
$$

where $\mathrm{W}^{\mathrm{nk}}$ is an $\mathrm{N}$ by $\mathrm{N}$ matrix with the element in the $n, k$ position, $W_{n, k}=\left(e^{-j 2 \pi / n}\right)^{n k}$. The $F F T$ is a procedure for factoring the $\mathrm{N}$ by $\mathrm{N}$ matrix $\mathrm{W}^{\mathrm{nk}}$ in such a

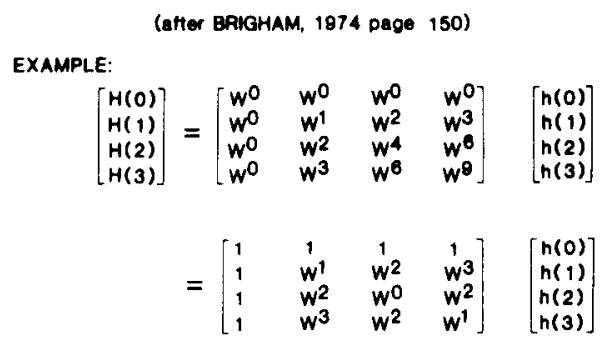

NOW FACTOR THE MATRIX

$\left[\begin{array}{l}H(0) \\ H(1) \\ H(2) \\ H(3)\end{array}\right]=\left[\begin{array}{cccc}1 & w^{0} & 0 & 0 \\ 1 & w^{2} & 0 & 0 \\ 0 & 0 & 1 & w^{1} \\ 0 & 0 & 1 & w^{3}\end{array}\right]\left[\begin{array}{cccc}1 & 0 & w^{0} & 0 \\ 0 & 1 & 0 & w^{0} \\ 1 & 0 & w^{2} & 0 \\ 0 & 1 & 0 & w^{2}\end{array}\right]\left[\begin{array}{l}h(0) \\ h(1) \\ h(2) \\ h(3)\end{array}\right]$

THE PRODUCT IS NOW CARRIED OUT IN TWO STEPS

$\left[\begin{array}{l}h_{1}(0) \\ h_{1}(1) \\ h_{1}(2) \\ h_{1}(3)\end{array}\right]=\left[\begin{array}{cccc}1 & 0 & w^{0} & 0 \\ 0 & 1 & 0 & w^{0} \\ 1 & 0 & w^{2} & 0 \\ 0 & 1 & 0 & w^{2}\end{array}\right]\left[\begin{array}{l}h(0) \\ h(1) \\ h(2) \\ h(3)\end{array}\right]$

AND
$\left[\begin{array}{l}H(0) \\ H(2) \\ H(1) \\ H(3)\end{array}\right]=\left[\begin{array}{l}h_{2}(0) \\ h_{2}(1) \\ h_{2}(2) \\ h_{2}(3)\end{array}\right]=\left[\begin{array}{llll}1 & w^{0} & 0 & 0 \\ 1 & w^{2} & 0 & 0 \\ 0 & 0 & 1 & w^{1} \\ 0 & 0 & 1 & w^{3}\end{array}\right]\left[\begin{array}{ll}h_{1}(0) \\ h_{1}(1) \\ h_{1}(2) \\ h_{1}(3)\end{array}\right]$

Figure 2. Schemata representing the reduction in calculations for the FFT over the FT where $N=4=2^{2}$.

manner as to minimize the number of multiplications and additions. For $\mathrm{N}=2^{\mathrm{r}}$, $\mathrm{W}^{\mathrm{nk}}$ is factored into $\mathrm{r} \mathrm{N}$ by $\mathrm{N}$ matrices. The example in Figure 2 for the case in which $\mathrm{N}=4=2^{2}$ illustrates the procedure and the reduction in the number of required calculations.

Note that the original procedure requires $\mathrm{N}^{2}$ multiplications and $\mathrm{N}(\mathrm{N}-1)$ additions (16 and 12 , respectively, for the example), whereas in the second procedure, after factoring, each step requires $N / 2$ multiplications and $\mathrm{N}$ additions for a total of $\mathrm{Nr} / 2$ multiplications and $\mathrm{Nr}$ additions ( 4 and 8 for the example). This results in a considerable savings in computer time over the calculations required in the FT.

\section{GEOMETRIC REPRESENTATION OF FFT}

In our electrophysiological experimentations, we find that an FFT fits our research needs, computer capacity, and time constraints better than an FT. The FFT in and of itself does not yield information that is directly usable and analyzable. It is the Fourier spectrum (FS) that is obtained from the FFT that ultimately yields usable data (Blackman \& Tukey, 1959; Nussbaumer, 1981). The FS is a breakdown of the power in the original data into its individual spectra or component parts. As in the optical analogy presented earlier, the beam of light is of a known total intensity, but until it is broken into its individual parts (colors), there is no direct way of telling which colors are present or how much of each color is present. The FFT takes the voltage vs. time data and transforms them into the aggregate of all the frequencies found in the data. 
The FS, which is derived from the FFT, indicates the amount of each frequency that is present in the aggregate of the data (Bracewell, 1978).

As an example of the application of FFT and FS to electrophysiological research, suppose that we took a 1 -sec sample of EEG data (voltage vs. time) from a sleeping and dreaming subject as shown in Figure 3. Let us assume that these data were gathered using an algorithm in which 256 data points were sampled each second. These data resemble what an investigator often obtains as theta-band data. Before these data can be submitted to a Fourier analysis, the data must first be converted from analog data to digital data (A/D conversion). This can be done using any of a number of different computer programs. If the digital data were submitted to an FFT, then the aggregate or total power of the neurons that fired in the time period could be obtained. If these data were further broken down using an FS, then an investigator would be able to tell the amount of the total power that is attributable to each of the $1-\mathrm{Hz}$ frequency bands. Figures 3, 4, and 5 are a series of graphs that give a geometric representation of how an FS is performed. Initially, a computer program is used to measure the amplitude of the wave that was obtained as the 1-sec sample of data. These are the 256 digital values that are obtained from the A/D conversion. These values are actually the lengths of the vertical lines that are labeled 1, 2, 3, 4 through 256 in the portion of Figure 3 labeled "Analog Signal." Approximately half of these 256 values are positive, and
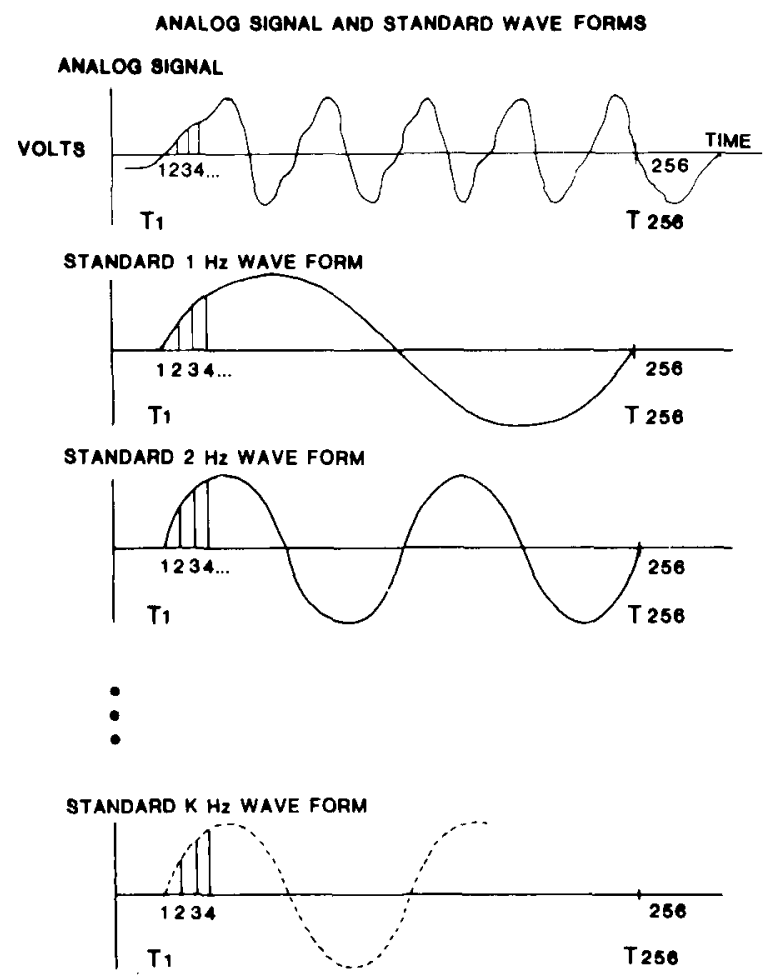

Figure 3. Geometric (pictorial) representation of the operations of the FFT for breaking a complex wave into its component parts.
DIGITIZED AND STANDARD WAVE FORM AMPLITUDES

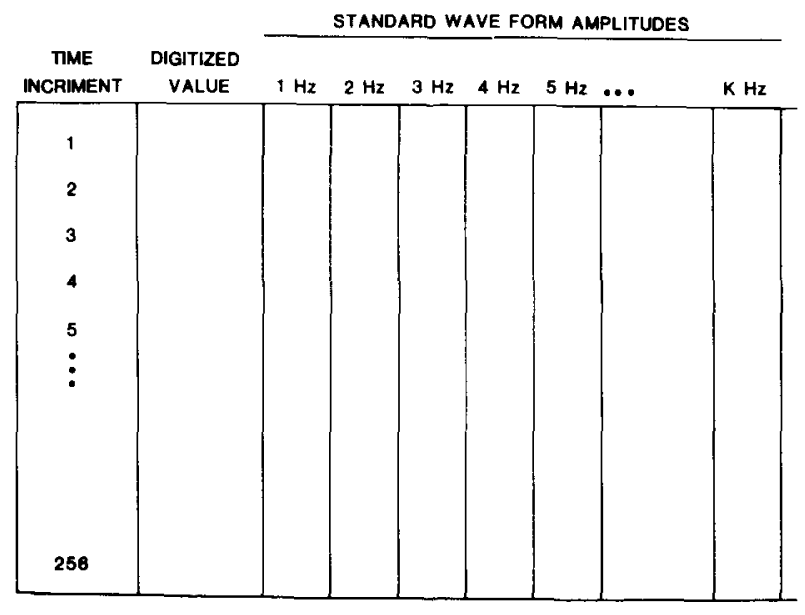

Figure 4. An analog of the numerical output of the FFT.

FOURIER SPECTRUM

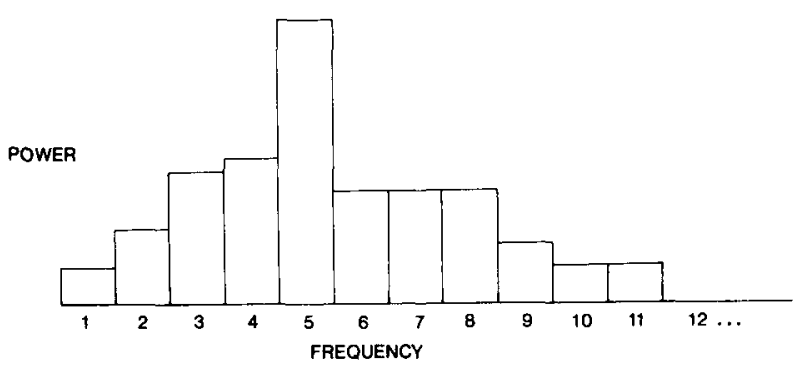

Figure 5. Typical representation of the Fourier spectra of the FFT output represented in Figure 4.

the other half are negative. Some of the values are or will approach zero. In actuality, this is a vector of 256 scores and would be recorded in Figure 4 in the column headed "Digitized Value." Once the digitized values of a sample wave are obtained, a set of amplitudes for a series of standard waves must be obtained. In the lower portion of Figure 3 are samples of $1-\mathrm{Hz}, 2-\mathrm{Hz}$, through $\mathrm{K} \cdot \mathrm{Hz}$ standard waves. The choice of the number of standard waves and their wavelengths is a function of the particular study that an investigator is conducting. Once the decision has been made as to which of the $1 . \mathrm{Hz}$ bands an investigator is interested in, the amplitude of the waves is found for each of the 256 data points. The sampling procedure used with each of these standard waves is identical to that which was carried out on the sample wave. The standard waves are constructed in integer frequencies, so that the amplitude of any one standard wave is orthogonal to or independent of all the other standard waves. If, as in our geometric example, we are interested in the first through the fifth $1-\mathrm{Hz}$ bands, then the 256 amplitudes of each of these standard waves would be placed in the appropriate columns of Figure 4. The data that would normally appear in Figure 4 are still expressed as a time-dependent function. The process of transforming the sample wave data of a 
time-dependent to a frequency-dependent function can be viewed as a type of correlational procedure. The procedure is one of finding the proportion of the variance of the sample wave that can be accounted for by each of the standard waves. If an investigator is interested in five $1-\mathrm{Hz}$ frequencies, then he calculates five coefficients. Each coefficient indicates the power (voltage squared) at each of the five frequencies for the $1 \mathrm{sec}$ for which the data were sampled. The power at each frequency is analogous to the correlation between the 256 data points from the sample waves and the 256 data points for each of the standard waves. Another way of describing this process is to state that the power at each frequency is a mathematical expression of how well the standard waves for each frequency fit the sample wave. The better the standard wave fits the sample wave, the higher the coefficient and the higher the power. The converse of this relationship also holds. That is, the poorer the fit of a standard wave is to the sample wave, the lower the power.

A histogram of the power at each of the frequencies for the sample wave is shown in Figure 5. Note that most of the power of the sample wave is at $5 \mathrm{~Hz}$ and that the original sample wave basically resembled thetawave data, of which $4 \mathrm{~Hz}$ or $5 \mathrm{~Hz}$ is the dominant frequency. Since the standard waves were constructed to be orthogonal or independent, the power at each of the $1-\mathrm{Hz}$ frequency bands is also orthogonal or independent.

\section{SUMMARY}

In our example, we started with an analog signal, converted it to a digital signal, and used an FFT and FS to reduce the data to the amount of power observed at each of a number of frequencies. This process is also reversible, and an investigator can take the data from the power of each of a number of frequencies and find the total power that was put out by the individual at the time that the individual's EEG data were gathered.

Along with the fact that the numeric values for each of the bands of an FS that are obtained are informative, they have another desirable characteristic. As a result of the mathematical algorithm that is used in deriving the data, the data are orthogonal or independent. This means that the amount of power in any one of the spectral bands is independent of the amount of power in any one or more of the other spectral bands. Another way of describing this is to say that there is no correlation among the data in any of the spectral bands. This means that, should an investigator be interested in the power in more than one of the spectral bands, the data will easily lend themselves to multivariate statistical analysis (Bremner et al. 1982). Although a multivariate statistical approach does not require that the criterion variables be independent, having them be orthogonal does make for a much cleaner statistical analysis.

There is a vast amount of hardware and software that is commercially available today that can be used in electrophysiological research. This includes many different computer programs to perform $A / D$ conversions, FFT, and FS (Eddy \& Bremner, 1983). It has been our finding that most investigators have their "favorite" program. "Favorite" is typically defined as the program that is available, and the one that the investigator knows how to run. Some run more efficiently than others, and some give output that is more complete and easily interpreted than others, but an investigator should have little trouble finding usable software (Eddy \& Bremner, 1983). The decision as to which FFT and FS program to purchase and use is a critical one. If an investigator chooses and implants a program that is easily run, that operates efficiently, that operates effectively, and that gives the appropriate output, then his research will move along very smoothly. If any of these four criteria are not met, then his research will become more difficult to conduct than it should be.

\section{REFERENCES}

Benignus, V., \& Muller, K. E. Information flow in the brain: Computer requirements (A tutorial). Behavior Research Methods \& Instrumentation, 1982, 14, 294-299.

Blackman, R., \& TukEY, J. Measurement of power spectra. New York: Dover, 1959.

Bracewell, R. N. The Fourier transform and its applications. New York: McGraw-Hill, 1978.

Bremner, F. J., Yost, M., \& McKenzie, R. Computer-managed inferential statistical analysis of EEG data. Behavior Research Methods \& Instrumentation, 1982, 14, 300-302.

Brigham, E. O. The fast Fourier transform. Englewood Cliffs, N.J: Prentice-Hall, 1974.

Eddy, D. R., \& Bremner, F. J. Computer packages that contain the FFT. Behavior Research Methods \& Instrumentation, 1983, 15, 254-257.

Glass, G. V., Wilson, V. L., \& Gotrman, J. M. Design and analysis of time-series experiments. Boulder: Colorado Associated University Press, 1975.

Nussbaumer, H. J. Fast Fourier transform and convolution algorithms. Berlin: Springer Verlag, 1981.

WALTHER, D. O. Spectral analysis for electroencephalograms: Mathematical determination of neurophysiological relationships from records of limited duration. Experimental Neurology, 1963, 8, 155-181. 